
\title{
The Counseling Psychologist
}

\section{Autonomy as Process and Outcome: Revisiting Cultural and}

\section{Practical Issues in Motivation for Counseling}

Martin F. Lynch, Maarten Vansteenkiste, Edward L. Deci and Richard M. Ryan

The Counseling Psychologist 2011 39: 286 originally published online 15

December 2010

DOI: $10.1177 / 0011000010388424$

The online version of this article can be found at:

http://tcp.sagepub.com/content/39/2/286

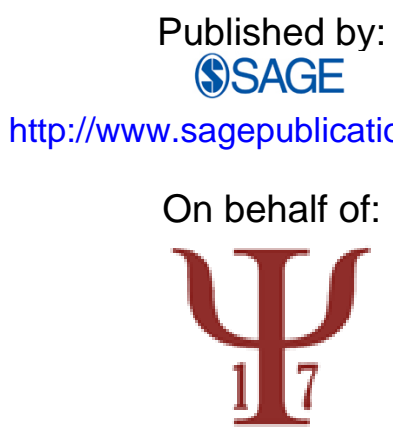

Division of Counseling Psychology of the American Psychological Association

Additional services and information for The Counseling Psychologist can be found at:

Email Alerts: http://tcp.sagepub.com/cgi/alerts

Subscriptions: http://tcp.sagepub.com/subscriptions

Reprints: http://www.sagepub.com/journalsReprints.nav

Permissions: http://www.sagepub.com/journalsPermissions.nav

Citations: http://tcp.sagepub.com/content/39/2/286.refs.html 
>> Version of Record - Jan 6, 2011

OnlineFirst Version of Record - Dec 15, 2010

What is This? 


\title{
Autonomy as Process and Outcome: \\ Revisiting Cultural \\ and Practical Issues \\ in Motivation for \\ Counseling
}

The Counseling Psychologist

39(2) 286-302

(c) 20II The Author(s)

Reprints and permission: http://www. sagepub.com/journalsPermissions.nav

DOI: $10.1177 / 0011000010388424$ http://tcp.sagepub.com

(SAGE

\section{Martin F. Lynch,' Maarten Vansteenkiste, ${ }^{2}$ Edward L. Deci,' and Richard M. Ryan'}

\begin{abstract}
Three commentators (Carter, 20II; Kim, 20II; Scheel, 20II) concurred with a central proposition of the target article (Ryan, Lynch, Vansteenkiste, \& Deci, 20 I I): that client motivation for counseling is of critical importance to counselors and therapists. In this Reply, we acknowledge and address a number of issues raised by the commentators, including the role of motivation and autonomy in multicultural counseling, the issue of common factors in counseling, and how the continuum of motivation proposed in the target article relates to the experience of practitioners who are engaged with a wide variety of client presentations. We maintain that the autonomous motivation of clients is a legitimate focus in counseling, both as process and outcome, and that an autonomy-supportive stance on the part of the counselor is implicit in the ethical mandate to respect the person of the client.
\end{abstract}

\section{Keywords}

motivation, autonomy, psychotherapy, ethics, multiculturalism

\footnotetext{
'University of Rochester, NY, USA

${ }^{2}$ Ghent University, Belgium

Corresponding Author:

Martin Lynch, Warner School of Education \& Human Development, University of Rochester,

P.O. Box 270425, Rochester, NY 14627

Email: mlynch@warner.rochester.edu
} 
We wish first to thank our commentators for their insightful remarks. We value such an exchange of ideas, not least because it provides us the opportunity to clarify and critique our own thinking about the role of client motivation in counseling. Accordingly, we take advantage of this opportunity to respond to several issues raised by the commentators and in the process to elaborate on points we introduced in our target article (Ryan, Lynch, Vansteenkiste, \& Deci, 2011 [this issue]).

\section{The Importance of Motivation in Counseling: Both Quality and Quantity}

We begin with a point on which we wholeheartedly agree: We are particularly gratified that the commentators concur with our assessment concerning the central role played by client motivation in the counseling endeavor. As we stated in the target article, without motivation, nothing happens. At the same time, we wish to emphasize the general point that, in our view, it is more informative for counselors to be interested in the quality than in the quantity of their clients' motivation. This point refers to the important distinction made within self-determination theory (SDT) between intrinsic and extrinsic motivation and the continuum of extrinsic motivations that we described in the target article and that ranges from external regulation to full volition. In other words, we argue on theoretical and empirical grounds that the client whose motivation for counseling is more highly integrated (i.e., autonomous) is likely to persist in therapy longer and derive more benefit from whatever time is spent in therapy than is the client whose level of motivation (in terms of sheer quantity) may be equally high, but in quality is highly externally regulated (i.e., controlled). This is because we expect that changes embraced by clients who are more autonomously motivated are more likely to be selfselected, congruent with the client's values, maintained over time, and generalized to life situations outside the counselor's office. This is not to suggest that change is easy, whether motivated by autonomous or controlled reasons. Change of any sort, whether in affect, cognition, or behavior, is likely to be difficult, and the processes required to achieve the hoped-for change may not be inherently pleasant or enjoyable (Vansteenkiste \& Sheldon, 2006), as also recognized by Carter (2011 [this issue]). Yet, we believe that change is likely to be deeper and longer lasting when it reflects the client's true assent to treatment rather than when change essentially proceeds from contingencies set up within and by the environment. The degree to which clients are, can become, or should become autonomous in their motivation for counseling is of course another question, one to which we will return. 
In this regard, the commentators have raised some very important issues that we will address, including the role of motivation and autonomy in multicultural counseling, the issue of common factors in counseling, and how the continuum of motivation relates to the experience of most practitioners, who are engaged with a wide variety of client presentations. Ultimately, these issues all have to do with the role of motivation as both process and outcome in counseling.

\section{Motivation in Multicultural Counseling}

Kim (2011 [this issue]) raised several important issues pertaining to the understanding of motivation from the perspective of multicultural counseling. These issues include the external validity of the motivation continuum in non-Western countries, the notion of enculturation, the problem of client/ therapist (mis)match, and the importance of a shared worldview. Before addressing these issues, we wish to convey that we agree that how counselors approach the motivation of clients whose backgrounds differ from their own in substantial ways, including but not limited to culture, is of central importance. Indeed, it goes to the heart of how we as counselors understand the nature of the client-counselor relationship and the process of counseling itself. In essence, it is about respect for the person of the client, a fundamental ethical value embraced by those in the helping professions. As we argued in the target article (Ryan et al., 2011), this principle of respect for the person of the client is grounded in longstanding philosophical discourse about the nature and universality of autonomy, a point to which we will return. In addition, we appreciate that underlying Kim's (2011) reflections is a deep concern that clients of non-Western cultural backgrounds not be a priori alienated or excluded from participation in counseling because counselors unreflectively implement techniques and theories grounded in worldviews that are at odds with the clients' worldviews. We echo that concern, because any such imposition is itself an infringement on autonomy.

\section{External Validity of the Continuum of Motivation}

Kim (2011) questioned whether the continuum of motivation, which is derived from SDT's taxonomy of motivation, applies equally to clients whose cultural worldviews are more collectivistic. Implicit in this question is the assumption that autonomy may be more relevant for clients whose worldviews are individualistic, a position taken by some cross-cultural psychologists (e.g., Markus \& Kitayama, 1991). Indeed, it is sometimes 
maintained that autonomy is important only for those whose personal beliefs, whether derived from the culture or from other sources, explicitly value autonomy. Thus, it would not be relevant for those whose values do not endorse autonomy. We welcome the opportunity to clarify our perspective on this issue.

We wish, first, to reiterate that we strongly distinguish autonomy from either independence or individualism (e.g., Chirkov, Ryan, Kim, \& Kaplan, 2003; Ryan \& Deci, 2006; Vansteenkiste, Zhou, Lens, \& Soenens, 2005). Although a dictionary definition might equate autonomy with independence, we draw our understanding from the phenomenological (e.g., Husserl, 1980; Pfander, 1908/1967; Ricoeur, 1966) and analytic (Dworkin, 1988; Frankfurt, 1969) traditions of philosophy to emphasize volition or self-rule. In other words, when autonomous, one's behaviors and beliefs are experienced as emanating from oneself and being congruent. This view includes the possibility that people would be autonomous with respect to a behavior or belief if they give their assent to it, even if the behavior or belief ultimately originates from an authority outside themselves. A classic example involves conformity to the traffic laws of the jurisdiction in which one resides: To the extent that I believe in the value of traffic laws - to the extent that I value the protection of my safety and the safety of others - it does not represent a violation of my autonomy to obey those laws, or to stop, when an officer of the law tells me to stop. Autonomy is not defined by where the command, edict, or norm is initiated but rather by one's freely giving assent. In this regard, one can understand the importance of distinguishing between the idea of autonomy as it is embodied in the continuum of motivation and the idea of independence that is implicit in cultural worldviews such as individualism.

Consistent with the distinction between autonomy and independence, the continuum of motivation refers to the degree to which one's motivation for a particular behavior, value, or belief has been internalized and integrated into oneself. Does one enact a particular behavior or hold a particular belief because one feels pressured and controlled (e.g., to avoid punishment or obtain a reward) or because one has self-endorsed the behavior or belief and thus is autonomous with respect to it (i.e., because one reflectively assents, having judged it to be congruent with one's integrated sense of self)? It is important to understand that the degree of internalization is separate from the actual content of the behavior or belief being internalized. This is illustrated by the fact that one person might enact a behavior for reasons that are very controlled, whereas another person might enact the same behavior for reasons that are autonomous. Indeed, the same person could enact the same behavior for controlled reasons in one situation and for autonomous reasons 
in another. The continuum, in other words, is content free, situation specific, and dynamic: The quality of motivation, resulting from the degree of internalization, changes depending on the person's prior experiences as well as on the phenomenal experience in the moment. Autonomy in this sense represents the mode or manner with which one embraces particular cultural content, or in motivational terms, the degree to which one has internalized that content. In technical terms, autonomy and independence are orthogonal: It is possible for one to be autonomously dependent on others (just as it is possible for one to be heteronomously independent), and indeed, some research suggests that autonomous dependence-being able to willingly rely on others - can be an important contributor to wellness (e.g., Ryan, La Guardia, Solky-Butzel, Chirkov, \& Kim, 2005; Ryan \& Lynch, 1989).

This analysis of the continuum of motivation allows us to assert, on theoretical grounds, that a person could espouse collectivistic beliefs and practices for reasons that are either more autonomous or more controlled; similarly, a person could espouse individualistic beliefs and practices for reasons that are either more autonomous or more controlled. Indeed, there is some empirical evidence suggesting that this is, in fact, the case. In studies involving participants from Turkey, Russia, Korea, Brazil, the United States, and Canada, Chirkov and his colleagues (Chirkov et al., 2003; Chirkov, Ryan, \& Willness, 2005) used Triandis's (1996; Triandis \& Gelfand, 1998) cultural model and found that the degree of internalization of cultural beliefs and practices was indeed separable from the content of those beliefs and practices: Both individualism and collectivism could be embraced for reasons that were more controlled or for reasons that were more autonomous. The same was true for the vertical and horizontal dimensions in Triandis's taxonomy. Across samples, however, the greater the individual experienced autonomy in enacting his or her cultural practices, the greater the well-being. There was no moderation by culture in the magnitude of this relation. It is interesting that results also suggested that it was easier for people to internalize horizontal beliefs and practices than vertical ones. This in itself may have interesting implications for counseling that go beyond our present discussion.

In light of the above, we believe that the taxonomy provided by the continuum of motivation can in principle apply to clients from diverse cultural backgrounds, whether collectivistic, individualistic, horizontal, or vertical. Of course, how counselors apply this framework with each particular client requires sensitivity as well as awareness of their own, perhaps culturally loaded, biases; it requires respect for the person of the client. But the point we wish to underscore is that we do not think the existence of cultural differences, per se, invalidates the potential usefulness of the continuum in 
one's clinical work with clients from other cultures. Indeed, to the extent that support for autonomy represents a universal ethical imperative to respect the person, values, and beliefs of each client—-beliefs and values that may fall anywhere along the spectrum from individualistic to collectivistic and from horizontal to vertical-we believe that an autonomy supportive attitude on the part of the counselor may be the best safeguard against cultural insensitivity.

Certainly, we in no way suggest that counselors do seek to disregard, much less to override, their clients' values and beliefs. Nor do we suggest that counselors should manipulate clients into valuing some practice or outcome. To the contrary, we consider it part of the counselor's ethical responsibility to assist clients, first, to identify what their own values, beliefs, and goals are and, having done so, to make an informed decision about whether to keep or modify them. A similar point could be made with regard to the experience and expression of emotion. Ultimately, it is about assisting clients to take ownership of their lives. "Taking ownership" in this sense involves helping clients to examine their values and beliefs; to work to more fully internalize them (or indeed to abandon them) if they are operating as controls; to work toward congruence of their behaviors with their endorsed values and beliefs; and to allow greater awareness of their emotions if controlled motivations have blocked those feelings from awareness.

The continuum of motivation reflects an acknowledgment that not all values or behaviors are intrinsically motivated and that it is possible to internalize values and behaviors that originate from sources outside oneself in such a way that one is still autonomous with respect to them, rather than unreflectively and slavishly enacting them. We believe that assisting clients to move in this direction is an ethical responsibility of the counselor based on the universal nature of autonomy as a principle, as we asserted in the target article (Ryan et al., 2011). This bears some similarity to the idea that the aim of Freudian analysis is ultimately about the liberation of the analysand by making what was unconscious conscious and, to the aim of Ellis's Rational Emotive Behavior Therapy (REBT), to liberate the client from the tyranny of the "should" which we refer to as the client's introjects, in terms of the motivation continuum).

This leads, however, to another important question raised by Kim (2011). Is there a value judgment implied in assessing where along the continuum a client's motivation for counseling is currently located? If the client's motivation is determined to be "not autonomous enough," ought that to elicit criticism (voiced or unvoiced) on the counselor's part? We might pose a parallel question: If the client is determined to be not healthy enough, ought that to elicit criticism (voiced or unvoiced)? We pose the question this way because 
greater autonomy has consistently been linked empirically to psychological health, across genders, ages, socioeconomic levels, and cultures. It is clear that the answer to both questions would be "no, the counselor ought not to be critical" in a judgmental sense, even though we do believe that it is appropriate for counselors to work with the aim of having clients become more autonomous and healthy.

We believe that the whole point of autonomy support on the part of counselors is to support clients examining their own values, emotional experiences, and behaviors precisely so they can make their own choices about accepting and integrating these values, emotional experiences, and behavioral regulations. Thus, we would consider it a violation of the principle of autonomy support to openly (or secretly) place a negative valuation on any values or beliefs endorsed by clients, including those that are externally motivated or introjected. Rather, in line with a humanistic perspective, we believe the appropriate clinical attitude with respect to virtually any client revelation is one of nonjudgmental interest and appreciation. At the same time, counselors would support their clients in examining these revelations and understanding their meaning for themselves. Thus, in contrast to some outcomefocused approaches, we would not exclude clients from participation in treatment because their motivation for counseling does not match some hypothetical "gold standard" or ideal about motivation. Instead, low volition for change suggests important issues to be grappled with, and that itself is a critical part of the process of counseling.

\section{Enculturation, Matching, and Motivation}

Kim (2011) called attention to the phenomenon of enculturation, according to which some potential clients might avoid counseling out of a desire to avoid bringing shame to the family. In other words, some culturally embedded values might appear antithetical to participation in counseling, to the extent that acknowledging a personal problem, whether emotional or behavioral, might reflect poorly in the eyes of one's community, on one's relatives, or on one's upbringing. Similarly, as Kim observed, when clients perceive a mismatch between their cultural beliefs about, for example, the expression of emotion and what they perceive to be the focus of a particular therapeutic approach, they may experience discomfort or even reject further treatment.

These are legitimate concerns to which the culturally sensitive counselor would attend. We might suggest that in the situation of the client for whom enculturation poses a problem, the counselor might respond empathically, recognizing that even coming to counseling, or disclosing within its 
confines, may be very difficult for the client. The counselor might explore with the client the nature of the barriers, conflicts, and risks, and what it was that led him or her to give counseling a try. With the client who is uncomfortable with personal disclosure, the counselor might explore nonjudgmentally the nature of the client's beliefs about emotions, and what culture and family mean for him or her in this regard. Motivationally speaking, we might find that some clients' reasons for not participating in counseling reflect a kind of controlled non-engagement, to the extent that they feel their acceptance by their group prevents them from seeking a counselor's help. In contrast, a focus on interpersonal harmony, for example, between client and counselor, might on the surface appear to be a positive thing, but counselors should be aware that such "harmony" may in reality reflect a kind of controlled compliance, that is, a relinquishing on the client's part of his or her personal values and beliefs on the assumption that this will make the client more "acceptable" to the counselor. Needless to say, this kind of compliance would not, generally speaking, be ideal within the motivational framework we propose (and might even reflect an underlying perception of conditional regard; see Assor, Roth, \& Deci, 2004). Ultimately, the idea is to respect the client's capacity to define what is best in an atmosphere free of pressure and evaluation, in which the relevant issues can be reflected upon.

\section{Shared Worldviews and Motivation}

In a related vein, Kim (2011) astutely recognized the potential importance of a shared worldview. The client who perceives or experiences that the counselor shares similar beliefs in some domain considered by the client to be important (e.g., cultural beliefs or values) is likely to feel a greater sense of connection with the counselor and to feel more understood by the counselor. These experiences are likely to be helpful in facilitating the client's volitional engagement in the counseling process. We agree with Kim that a shared worldview is likely to facilitate the alliance between counselor and client and to have important benefits for client motivation.

We would not go so far as to suggest that a shared worldview is a prerequisite for the counseling relationship. Again, a truly autonomy-supportive stance will allow the counselor to enter into the client's worldview in an accepting, interested, nonjudgmental way, and it is this stance, we believe, that will ultimately enhance both the alliance and client motivation. This leads us to the insights offered by Scheel (2011 [this issue]). 


\section{Autonomy and the Alliance: Common Factors in the Counseling Process}

Scheel (2011) called attention to the fact that client motivation, presented in the target article (Ryan et al., 2011) in terms of the construct of autonomy, may prove to be as important a common factor across effective therapies as is the alliance between client and counselor. We agree, and a similar suggestion was offered by Zuroff et al. (2007). Indeed, in the Zuroff et al. research, client autonomy was found to account for more variance in remission of depression than was therapeutic alliance. Furthermore, both client motivation and therapeutic alliance were predicted by the therapists' autonomy support across three different therapeutic approaches. In the target article (Ryan et al., 2011), we argued that virtually all therapeutic schools attend to the issue of client motivation and, more to the point, that they acknowledge either explicitly or implicitly that what we call autonomous motivation is the preferred form of client motivation.

Scheel (2011) raised a very good point. Client motivation and the working alliance between client and counselor go hand in hand. It is indeed very likely that the two influence each other. Keeping in mind the distinctions we draw along the continuum of motivation, we would emphasize that when clients experience their counselors as autonomy supportive, they are likely, during the course of therapy, to become more autonomously motivated for counseling, even if their initial motivation was toward the controlled end of the continuum. Evidence suggests that even patients who have been court-mandated for treatment - in other words, whose initial motivation for treatment is likely to be highly external, that is, controlled - reported more autonomous motivation to the extent that they experienced their providers as supportive of their needs for autonomy and relatedness (Lynch, Plant, \& Ryan, 2005; Zeldman, Ryan, \& Fiscella, 2004).

As Scheel (2011) suggested, it is likely that client motivation also affects the nature of their alliance and the quality of their relationship with the counselor. For example, it may be hard for the counselor to remain autonomy supportive with a client who is reactive or who "pulls" for controlling interventions (perhaps even despite the counselor's best efforts to remain empathic and accepting toward all of his or her clients). We agree that there are likely to be reciprocal effects and influences, as Scheel suggested and as we have observed in other relational research on autonomy support (Deci, LaGuardia, Moller, Scheiner, \& Ryan, 2006) and on helping relationships (Weinstein \& Ryan, 2010).

Scheel (2011) suggested that clients are more likely to accept treatment when therapists' interventions are closely matched to the clients' strengths 
and their beliefs about change. Scheel further pointed out that research indicates that matching interventions are more likely to be implemented. This suggestion can be interpreted in at least two ways. In one interpretation, the point is really parallel to that made by Kim (2011) with regard to matching counselor and client cultural worldviews, and we agree that that could facilitate the counseling process. A second interpretation is that clients will more likely accept treatment if it matches their motivation for treatment. This would suggest that if clients were strongly controlled, counselors would be more effective by being controlling, that is, by matching their clients' motivation. We would not agree with that view, and we have not found any empirical evidence for it. Indeed, the evidence seems to indicate that counselors being autonomy supportive rather than controlling is likely to be more effective regardless of the clients' initial motivation. Accordingly, we would not suggest that successful counseling depends on matching, that is, we would not consider matching to be a necessary condition.

To introduce the motivational taxonomy we described in the target article, it would be interesting to study precisely how a client-counselor match, along various dimensions (beliefs about change; worldview; gender; ethnicity), affects not only the quantity of client motivation but the quality of client motivation. Are clients who experience a match with their counselor along some important dimensions likely to experience motivation that is more autonomous or possibly more controlled? Does the answer to this depend on other factors, such as the degree to which the client also experiences unconditional positive regard from the counselor? These are interesting and important empirical questions.

\section{Client Motivation in Real-World Practice}

Carter (2011) provided a critique of the target article from the point of view of the practitioner. As practitioners ourselves, we are very sympathetic to the idea that abstract ideas and conceptualizations need to have grounding in real-world practice. Our everyday experience suggests that not all such theories are equally practical or compelling when set into the field.

One of the issues raised has to do with what the counselor's assumptions are concerning clients' motivation when they pick up the phone to schedule their first appointment. Clinicians from some theoretical traditions might well assume that the client is, or needs to be, highly motivated before beginning counseling. We do not. As we emphasized in the target article, we consider the quality of the client's motivation, that is, whether it is more autonomous or controlled, to be the most interesting and meaningful motivational issue, both 
when counseling begins and as it unfolds. It is true, however, as we stated earlier, that if clients approach counseling for reasons that are more autonomous, they are more likely to persist in the difficult task of change and to maintain and generalize their changes outside of the counseling office. Yet, as we also pointed out, we do not consider autonomous motivation a prerequisite for counseling, and we certainly agree with Carter (2011) that it may well be unrealistic to assume that a given client will find the process of counseling to be enjoyable (see, e.g., Vansteenkiste \& Sheldon, 2006). Indeed, enjoyment is only one component of autonomous motivation, as one can also freely assent to something that one finds unenjoyable, difficult, or even painful. This is in part the genesis of the idea that extrinsic motivation can in some situations be autonomous. Such assent is, of course, easier to give, to the extent that one finds the thing to which one is assenting personally important or meaningful.

As Carter (2011) pointed out, some clients enter counseling not clearly motivated for change. All they know is that they are in pain and they want the pain to end, and whether this motivation to end the pain is more internal or external may well not be their primary concern. The analogy of "holding" the client's motivation at such times, suggested by Carter, may be a useful one. Still, clients in pain are not likely to be amotivated for counseling. Hence, during the process of helping clients articulate what it is they would like to change in order to lessen the pain, their motivation for change may become clearer. In other words, it may become clear whether they are there because they were pressured to attend or alternatively because they personally believed that therapy would be helpful. As well, they may have had some vague sense that they would benefit from a particular kind of relationship - a supportive relationship - or that there are some goals that they would like to be able to accomplish. Any of these matters - feeling pressured versus free, feeling a desire for a nurturing and accepting relationship, or having some vague goals for one's life-could provide the counselor's entry point for exploring with the client, perhaps delicately, but certainly with interest and curiosity, the quality of the client's motivation for change. Furthermore, the process of exploration will likely lead to various emotions that the clients are dutifully suppressing, or perhaps ruminating about, and these can provide the basis for examining one's motivation for change. We believe that the classic opening question, "What brings you to counseling today?" ultimately implies an interest, not only in what the client hopes to get out of counseling but also in the nature of the client's motivation for change.

At this point, we would like to underscore that, although in our target article (Ryan et al., 2011) we argued that some theories of counseling consider client motivation to be a prerequisite of counseling and others consider 
it to be a process or perhaps an outcome, our own view is that motivation is the ongoing "stuff" of counseling, in line with more process-focused approaches. Furthermore, we agree with the ethical perspective, derived from existentialphenomenological philosophy, that autonomy may appropriately be conceived of as an end or outcome of counseling. So, for example, we would not necessarily be troubled by the client who, when becoming more autonomous about his or her participation in counseling, autonomously decided to drop out. Aside from this rather extreme case, however, we would in general consider that more autonomous reflection about one's life and more autonomous endorsement of any changes one might wish to make in one's life are a good thing, even something toward which the counselor can legitimately aim in the course of one's therapeutic work with the client.

Carter's (2011) thoughts about autonomy and valence require some response. We wish first to clarify that we do not consider the continuum of motivation to be a hierarchy of motivation. The continuum is intended to be descriptive of the degree to which a behavior is autonomous. That is, it is meant to help the clinician locate, in general terms, where the client's motivation is at any point during the counseling relationship, and with respect to the particular problem or issue with which the client is struggling in the moment. The continuum is also dynamic, reflecting the fluidity and flexibility of motivation, which changes in response to many factors, some coming from inside the client, others coming from outside the client, including the counselor's style or way of being with the client (e.g., more controlling, or more autonomy supportive). But it is not meant to suggest that external regulation is bad or that identified motivation is good, in an evaluative sense. It describes differences in autonomy, and the consequences of those differences are empirically, rather than subjectively, derived. The continuum is also not meant to suggest some kind of developmental, qualitative stage-based theory. We do not believe that motivation for any given activity must begin at the controlled end of the continuum and must progress toward the autonomous end of the continuum, although we have found empirically that most often greater autonomy is associated with greater health and wellness. And although we agree that internal motivation in its prototypical form - intrinsic motivationreflects the enjoyment of discovery and growth, our approach acknowledges and highlights that many if not most activities that we humans undertake we do for reasons that may in fact be only partly internalized. In other words, our motivation generally falls somewhere along the continuum from external, to introjected, to identified, to integrated, and may well not reflect the enjoyment characteristic of truly intrinsically motivated activity. But if we can give our assent to the activity, our motivation has moved that much closer toward the autonomous end of the continuum. 
As Carter (2011) points out, the case of the client who is feeling helpless or hopeless may be a special case worth thinking about, motivationally speaking, because it does not seem to fit easily within the continuum of motivation, as we have described it. Indeed, technically speaking, amotivation, as such, falls outside the motivational continuum, as it reflects the absence of motivation. How, then, can the counselor working with the amotivated client proceed? On what motivational grounds can the counselor stand, when the client arrives in the office either helpless or hopeless?

Working with the helpless or hopeless client is indeed likely to be a challenge, perhaps in part because we are likely to be confronted with our own desire to rescue, to provide what we think the client is lacking, which in this case is motivation. An important first step may be to clarify what we mean by stating that the client is helpless or hopeless. In some cases, it may be that clients cannot express a genuine desire for change, because they themselves do not have the language with which to express their desires, do not have a belief that their own desires are legitimate, or more to the point, do not have a sufficiently integrated sense of self (e.g., Rogers, 1961) because of early and chronic experiences of conditional regard from caregivers. In line with Rogerian thought, the task of the counselor in such situations is to help the client begin to validate his or her own inner experience through careful reflection and unconditional positive regard. Indeed, one of the tasks of the autonomy-supportive counseling relationship is to assist clients in identifying and validating their own inner experiences, emotions, and values.

But if we are speaking of the kind of helplessness and hopelessness that is sometimes associated with clinical depression, we are presented with a particular motivational challenge, although our response may not be all that different. Of course, in line with current best practices, we always want to consider whether a particular client might benefit from psychopharmacological intervention. And it is possible that the counselor's stance may at times resemble that of a coach, who instills hope in the hopeless or despondent client. But we encourage counselors to resist, to the extent possible, the urge to rescue their clients and, rather, to be with the clients in the midst of their pain, helping them find their own light at the end of their own tunnel. Helplessness is generally learned, and so the counselor's motivational task may be to help such clients identify small steps they can accomplish successfully, leading toward larger steps that will eventually help them break free of their self-evaluations of incompetence and the isolation that so often accompanies depression. In this way, the counselor in effect assists the client to counter the learned helplessness by learning to identify, and satisfy, the three basic psychological needs proposed by self-determination theory as essential in human motivation and wellbeing: the needs for autonomy, for competence, and for relatedness. 
It is clear that not all clients come to counseling autonomously motivated. Some come because they are mandated by legal authorities, they are pressured by a spouse or significant other, or they feel they "should" even though they themselves do not truly want to come. But can clients become more autonomous in their motivation, regardless of their starting point along the continuum of motivation? Ought we as counselors to make it our goal to assist clients toward autonomy? It is a curious property of autonomy support that, when genuinely enacted, it cannot result in pressuring clients to alter their worldview. It may invite them to explore their values and beliefs in ways that are new, unfamiliar, and therefore possibly uncomfortable. But it will always assist clients to get in touch with their own inner emotional experience, their own inner voice, and their own values, with the hope that clients will make their own authentic choices - whether to embrace the familiar (the roles and worldviews they have learned and, to greater or lesser extent, internalized) or to strike out toward new and unfamiliar territory. But if the autonomysupportive counseling relationship helps the client make his or her own authentic choice, we consider that a successful outcome, even if the choice differs from what the counselor might value or desire. In our view, autonomy support is implicit in the ethical mandate to respect the person of the client.

\section{Summary}

The three commentators have offered a diverse set of comments that have enriched the discussion of motivation in counseling and its relation to counselor autonomy support. We reiterate the central thesis of our target article, which is that all theories of counseling grapple either implicitly or explicitly with the problem of client motivation and autonomy, and they use various techniques to promote it. Becoming more differentiated in observing the various forms of motivation that clients can manifest, and the various ways in which counseling techniques and styles can influence these, we believe can help us to refine our methods and better ensure the engagement in and maintenance of changes that are made within the therapeutic process.

\section{Declaration of Conflicting Interests}

The authors declared no potential conflicts of interests with respect to the authorship and/or publication of this article.

\section{Funding}

The authors received no financial support for the research and/or authorship of this article. 


\section{References}

Assor, A., Roth, G., \& Deci, E. L. (2004). The emotional costs of perceived parental conditional regard: A self-determination theory analysis. Journal of Personality, $72,47-87$.

Carter, J. A. (2011). Changing light bulbs: Practice, motivation, and autonomy. The Counseling Psychologist, 39, 261-266.

Chirkov, V., Ryan, R. M., Kim, Y., \& Kaplan, U. (2003). Differentiating autonomy from individualism and independence: A self-determination theory perspective on internalization of cultural orientations and well-being. Journal of Personality and Social Psychology, 84, 97-110.

Chirkov, V. I., Ryan, R. M., \& Willness, C. (2005). Cultural context and psychological needs in Canada and Brazil: Testing a self-determination approach to the internalization of cultural practices, identity and well-being. Journal of Cross-Cultural Psychology, 36, 423-443.

Deci, E. L., LaGuardia, J. G., Moller, A. C., Scheiner, M., \& Ryan, R. M. (2006). On the benefits of giving as well as receiving autonomy support: Mutuality in close friendships. Personality and Social Psychology Bulletin, 32, 313-327.

Dworkin, G. (1988). The theory and practice of autonomy. New York: Cambridge University Press.

Frankfurt, H. G. (1969). Alternate possibilities and moral responsibility. The Journal of Philosophy, 66, 829-839.

Husserl, E. (1980). Ideas pertaining to a pure phenomenology and to a phenomenological philosophy-Third book: Phenomenology and the foundations of the sciences (T. E. Klein \& W. E. Pohl, Trans.). Dordrecht: Kluwer.

Kim, B. S. K. (2011). Client motivation and multicultural counseling. The Counseling Psychologist, 39, 267-275.

Lynch, M. F., Plant, R. W., \& Ryan, R. M. (2005). Psychological needs and threat to safety: Implications for staff and patients in a psychiatric hospital for youth. Professional Psychology, 36, 415-425.

Markus, H. R., \& Kitayama, S. (1991). Culture and the self: Implications for cognitions, emotion, and motivation. Psychological Review, 98, 224-253.

Pfander, A. (1967). Phenomenology of willing and motivation (H. Spiegelberg, Trans.). Evanston, IL: Northwestern University Press. (Original work published 1908)

Ricoeur, P. (1966). Freedom and nature: The voluntary and the involuntary (E. V. Kohak, Trans.). Chicago: Northwestern University Press.

Rogers, C. R. (1961). On becoming a person: A therapist's view of psychotherapy. Boston: Houghton Mifflin. 
Ryan, R. M., \& Deci, E. L. (2006). Self-regulation and the problem of human autonomy: Does psychology need choice, self-determination, and will? Journal of Personality, 74, 1557-1586.

Ryan, R. M., La Guardia, J. G., Solky-Butzel, J., Chirkov, V. I., \& Kim, Y. (2005). On the interpersonal regulation of emotions: Emotional reliance across gender, relationships, and culture. Personal Relationships, 12, 146-163.

Ryan, R. M., \& Lynch, J. (1989). Emotional autonomy versus detachment: Revisiting the vicissitudes of adolescence and young adulthood. Child Development, 60, 340-356.

Ryan, R. M., Lynch, M. F., Vansteenkiste, M., \& Deci, E. L. (2011). Motivation and autonomy in counseling, psychotherapy, and behavior change: A look at theory and practice. The Counseling Psychologist, 39, 193-260.

Scheel, M. J. (2011). Client common factors represented by client motivation and autonomy. The Counseling Psychologist, 39, 276-285.

Triandis, H. C. (1996). Individualism and collectivism. Boulder, CO: Westview.

Triandis, H. C., \& Gelfand, M. J. (1998). Converging measurement of horizontal and vertical individualism and collectivism. Journal of Personality and Social Psychology, 74(1), 118-128.

Vansteenkiste, M., \& Sheldon, K. M. (2006). There's nothing more practical than a good theory: Integrating motivational interviewing and self-determination theory. British Journal of Clinical Psychology, 45, 63-82.

Vansteenkiste, M., Zhou, M., Lens, W., \& Soenens, B. (2005). Experiences of autonomy and control among Chinese learners: Vitalizing or immobilizing? Journal of Educational Psychology, 97, 468-483.

Weinstein, N., \& Ryan, R. M. (2010). When helping helps: Autonomous motivation for prosocial behavior and its influence on well-being for the helper and recipient. Journal of Personality and Social Psychology, 98, 222-244.

Zeldman, A., Ryan, R. M., \& Fiscella, K. (2004). Client motivation, autonomy support and entity beliefs: Their role in methadone maintenance treatment. Journal of Social and Clinical Psychology, 23, 675-696.

Zuroff, D. C., Koestner, R., Moskowitz, D. S., McBride, C., Bagby, M., \& Marshall, M. (2007). Autonomous motivation for therapy: A new non-specific predictor of outcome in brief treatments of depression. Psychotherapy Research, 17, 137-148.

\section{Bios}

Martin F. Lynch, a clinical psychologist, currently serves as Assistant Professor in the counseling and counselor education programs at the Warner School of Education, University of Rochester. His research focuses on the effects of social context on human motivation, personality development, and well-being. His current research interests include cross-cultural issues in the role of autonomy support; the sources of 
within-person variability in trait self-concept, well-being, and life satisfaction; motivation for emigration; and adjustment of international students. He is also involved in applied motivational research in the domains of health care, education, work, and psychotherapy. Lynch, who has lived and worked in Russia, publishes in both western and Russian journals. Lynch is a member of both the American Psychological Association and the American Counseling Association.

Maarten Vansteenkiste is a faculty member in the Psychology Department at Ghent University in Belgium. A motivation psychologist, he has focused on expanding selfdetermination theory in new directions. For example, one thrust of his work has manipulated intrinsic relative to extrinsic goals on effective performance, social functioning, and psychological well-being. He has worked, both theoretically and empirically, on examining the differences and commonalities between self-determination theory and other well-established theories of motivation. His research and writings, which have been published in many top international journals, have also explored the effects of motivational regulations and goal contents in various life domains, including education, work and unemployment, psychotherapy, parenting, and sport and exercise.

Edward L. Deci is the Helen F. and Fred H. Gowen Professor in the Social Sciences at the University of Rochester. For 40 years Deci has been engaged in a program of research on human motivation, much of it with Richard M. Ryan, that has led to and been organized by self-determination theory. He has published ten books, including: Intrinsic Motivation (1975) and Intrinsic Motivation and Self-Determination in Human Behavior (co-authored with R. M. Ryan, 1985). A grantee of NIH, NSF, and IES, and a fellow of APA and APS, he has lectured at more than 90 universities around the world.

Richard M. Ryan is a Professor of Psychology, Psychiatry, and Education at the University of Rochester. He is a widely published researcher and theorist in the areas of human motivation, development, and psychological well-being, having published over 300 articles and books. He is a Fellow of several professional organizations including the American Psychological Association and the American Educational Research Association. Ryan is a licensed clinical psychologist with a practice in psychotherapy and consultation to schools and organizations, and has served as Director of Clinical Training at Rochester. Ryan is also an award-winning teacher and educational researcher, and is currently Editor-in-Chief of the psychological journal Motivation \& Emotion. 